\title{
ESTRATÉGIA, COMBINAÇÃO E RETICÊNCIAS ${ }^{1}$
}

\author{
Hellen Taynan Cavalcanti ${ }^{2}$ \\ Jonathan Simões Freitas
}

\begin{abstract}
RESUMO
Este trabalho tem como objetivo discutir teoricamente aspectos da Estratégia como processo e como prática social. Assim, consiste em um ensaio teórico que caracteriza ambas as ênfases de pesquisa e aponta possibilidades de combinação entre essas tradições. Nesse contexto, fornece uma visão crítica, que aproxima a abordagem micro da Estratégia como prática a uma análise sociológica, enquanto a Estratégia como processo é tida como associada às práticas de gestão na Administração e ao seu ideário centrado no desempenho organizacional. Dessa forma, o trabalho, apesar de revisar recentes propostas internacionais de diálogo entre essas tradições, acaba por destacar a possível incomensurabilidade de combinação direta dessas abordagens, devido aos diferentes valores e crenças subjacentes aos enfoques no mercado ou na sociedade como o contexto primário das organizações.
\end{abstract}

Palavras-chave: Administração. Estratégia como processo. Estratégia como prática.

\section{STRATEGY, COMBINATION AND SUSPENSION POINTS}

\begin{abstract}
This paper aims to theoretically discuss aspects of Strategy as a process and as a social practice. Thus, it consists of a theoretical essay that characterizes both research emphases and points out possibilities of combination between these traditions. In this context, it provides a critical view that approximate the micro approach of Strategy as practice to a sociological analysis, while Strategy as a process is considered to be associated with management practices in administration and its ideas centered on organizational performance. Thus, the work, despite reviewing recent international proposals for dialogue between these traditions, eventually highlights the possible incommensurability of direct combination of these approaches, due to the different values and beliefs underlying market or societal approaches as the primary context of organizations.
\end{abstract}

Keywords: Management. Strategy as a process. Strategy as a practice.

${ }^{1}$ Como citar este artigo: CAVALCANTI, H. T.; FREITAS, J. S. F. Estratégia, combinação e reticências. ForScience, Formiga, v. 8, n. 1, e00695, jan./jun. 2020. DOI: 10.29069/forscience.2020v8n1.e695.

${ }^{2}$ Autor para correspondência: Hellen Taynan Cavalcanti, e-mail: hellentaynan@gmail.com 


\section{INTRODUÇÃO}

Este trabalho tem por objetivo discutir teoricamente aspectos da Estratégia no contexto da estratégia como processo e como prática social. Quanto ao procedimento metodológico, esta pesquisa classifica-se como um ensaio teórico. Destaca-se, assim, que este trabalho parte de uma inquietação sobre a análise da Estratégia no campo da Administração. Embora literaturas internacionais sobre o tema tenham proposto uma combinação entre as abordagens da prática e as processuais (FLOYD et al., 2011; BURGELMAN et al., 2018; KOUAMÉ; LANGLEY, 2018), não existem publicações nacionais que explorem essa possibilidade (c.f. JARZABKOWSKI; KAPLAN, 2010).

Assim, este trabalho engaja-se com essas discussões, problematizando o viés integracionista que vem sendo proposto para combinar essas duas tradições da Estratégia. Especificamente, o ensaio, primeiramente, caracteriza as duas vertentes para contextualizar as soluções propostas para as suas divergências e, então, questionar se essas iniciativas de fato não ferem os princípios e valores internos de cada um desses dois programas de pesquisa. Em especial, destaca-se o risco de qualquer síntese promover um gerencialismo que perde a contribuição da reflexão crítica, ou uma visão redutível a um olhar sociológico que se distancia do fenômeno da gestão e da administração propriamente ditos. Diante desse cenário, propõese, ao invés de integrações abstratas artificialmente construídas, a adoção da metáfora das reticências como um modelo de abertura e não de achatamento das pesquisas em Estratégia. Dessa forma, promove-se um pluralismo que respeita a identidade de cada vertente de pesquisa, sem necessariamente descartar possibilidades de diálogo real a partir - e não a despeito - dessas identidades.

Afinal, estudos sobre estratégia estão longe do esgotamento, uma vez que, ao levar o tema para o campo da práxis, diversas disciplinas despertam o interesse de pesquisa. Desse modo, destacando que o ensaio teórico parte da inquietação dos pesquisadores diante de possibilidades ainda não exploradas, propomos reticências para o desenvolvimento futuro do campo da estratégia.

Em termos da estrutura deste ensaio, aborda-se, primeiramente, uma breve explanação sobre a importância da Estratégia em si. Posteriormente, apresentamos o pensamento de alguns autores que se posicionam em defesa da Estratégia voltada ao processo. Em seguida, são discutidos outros autores que buscaram aprofundar argumentos em defesa da Estratégia como prática social. Como eixo da terceira discussão, apresentamos a análise da combinação entre as duas abordagens propostas recentemente por Burgeman et al. (2018), dentre outros. Por fim, 
algumas considerações finais são tecidas, compartilhando reticências e ponderações sobre as possibilidades apresentadas.

\section{PROPOSTA DE UMA BREVE DISCUSSÃO TEÓRICA}

Neste tópico, apresentaremos uma proposta de discussão teórica, revisando alguns textos clássicos sobre diferentes abordagens da Estratégia relevantes para o tema deste trabalho.

\subsection{Por que a Estratégia é Importante?}

O legado seminal de Michael Porter para o campo de Estratégia não pode ser ignorado (ARGYRES; MCGAHAN, 2002; STONEHOUSE; SNOWDON, 2007). Esse autor definiu Estratégia como "a criação de uma posição ímpar e valiosa, envolvendo um conjunto diverso de atividades" (PORTER, 1996, p. 10). Contudo, deve-se pontuar que sua análise estava focada em fatores estruturais, atrelando condições características do ambiente ao desempenho organizacional - este sempre considerado de forma relativa ao de outras organizações, em termos de vantagem competitiva (GRECKHAMER, 2010). Esse entendimento faz com que a conduta estratégica seja determinada pelo contexto ambiental estando ligada a uma abordagem de estratégia-como-conteúdo (ECO), que enfatiza os fatores abstratos (i.e. as variáveis) que devem ser enfocados na formulação estratégica (HOSKISSON et al., 1999; GUERRASMARTIN; MADHOK; MONTORO-SANCHEZ, 2014).

Outra perspectiva também localizada nessa abordagem da ECO, mas que representa a principal alternativa à ênfase porteriana na análise do ambiente externo, é a chamada "visão baseada em recursos"(VBR) (SPANOS; LIOUKAS, 2001; HERRMANN, 2005). Essa perspectiva enfatiza a estrutura dos recursos internos à organização e não do seu setor, como os fatores efetivamente determinantes de seu desempenho sustentável. Os trabalhos dessa linha começaram na década de 1980, mas foram efetivamente legitimados a partir do clássico artigo de Barney (1991). Desde então, a VBR diferenciou-se em várias perspectivas de enfoque similar (FREITAS et al., 2017), com destaque para a ênfase em competências essenciais (PRAHALAD; HAMEL, 1990) e a teoria das capacidades dinâmicas, que visou a superação da análise estrutural estática de suas precedentes (TEECE; PISANO; SHUEN, 1997; TEECE, 2007).

Outra abordagem que se desenvolveu em paralelo à ECO foi a chamada "estratégiacomo-processo" (EPO) (HUFF; REGER, 1987; HUTZSCHENREUTER; KLEINDIENST, 
2006; MELLAHI; SMINIA, 2009). Comumente associada aos trabalhos pioneiros de Henry Mintzberg (MINTZBERG, 1978; MINTZBERG; WATERS, 1985), essa abordagem enfatizou, não as estruturas determinantes do posicionamento estratégico, mas, sim, os estrategistas e o processo de desenvolvimento da Estratégia ao longo do tempo (LANGLEY, 2007).

Incubada dentro dessa tradição, mas posteriormente rompendo com essas raízes processuais, foi estabelecida a abordagem da estratégia-como-prática, ECA (WHITTINGTON, 2007). Esta nasceu alinhada ao interesse da EPO em se aproximar da práxis da estratégia, distanciando-se das explicações estruturalistas representadas pelos porterianos e pela VBR (WHITTINGTON, 1996). Contudo, com o tempo, considerou que a EPO não chegou próximo o suficiente da atividade estratégica concreta (JOHNSON; MELIN; WHITTINGTON, 2003) e, por isso, propôs uma renovada concepção da Estratégia como um fenômeno social, em que atores instanciam, no cotidiano, práticas mais amplas da sociedade -em geral reproduzindo-as, mas podendo, também, reconfigurá-las (HITTINGTON, 2006; CHIA; MACKAY, 2007). Nesse sentido, seria a própria ortodoxia proposta nas escolas de administração e adotada por consultores que requereria que toda organização tenha uma estratégia (KNIGHTS; MORGAN, 1991). De fato, nesse artigo clássico dessa linha, Knights e Morgan (1991) argumentam que a questão crítica está no fato de que a ortodoxia das escolas e dos profissionais que elevaram a importância da Estratégiano contexto organizacional tratam o discurso da estratégia corporativa como uma característica natural, e não problemática. Neste sentido, os autores defendem, por exemplo, a necessidade de explorar o caráter problemático da Estratégia examinando a história que seria, em primeira e última análise, uma formação material-discursiva.

Diante desse breve panorama da evolução da pesquisa em Estratégia, este artigo prosseguirá para o detalhamento da EPO, seguido por uma apresentação da EPA, e concluindo com uma discussão dos futuros em aberto para esse campo, diante das relações possíveis entre essas tradições.

\subsection{Estratégia como Processo}

No que se refere à abordagem da Estratégia como processo (EPO), o trabalho de Sminia (2009) parece fornecer um ponto de partida satisfatório, uma vez que propõe uma revisão dos principais autores desta abordagem. A EPO dedica-se a responder a questões relativas ao “como" da Estratégia e, segundo Sminia (2009), Mintzberg foi um dos primeiros a lançar tal questionamento em relação à gestão. 
O estudo da EPO deu-se de forma a acompanhar estudos de caso longitudinais em organizações particulares, a fim de descrever a estratégia em um ambiente permeado por fluxos ao longo do tempo(e.g. BURGELMAN, 1996). Este posicionamento contribuiu para a elaboração de um conceito de Estratégia voltado a um processo de mudança (CHIA, 2002). Sminia (2009) destaca que tal entendimento levou Mintzberg e Waters (1985) a ver o processo como uma conjunção de estratégia pretendida e estratégia emergente. Neste esforço, havia a necessidade, apontada por Mintzberg de conduzir estudos de caso para abordagens mais descritivas que conduzissem à aproximação do que estava acontecendo. Sobre isso, havia fortes críticas, uma vez que, até então, as pesquisas estavam mais focadas a um caráter prescritivo.

O ponto focal na pesquisa de processos é a questão sobre se, e como, as estratégias de uma empresa são formadas ao longo do tempo (LECHNER; MÜLLER-STEWENS, 2000). A EPO concebe a Estratégia como formulação e implementação (HUTZSCHENREUTER; KLEINDIENST, 2006). Nesta dinâmica, a ação é o processo que visa à tomada de decisão e mudança organizacional de larga escala(WOLF; FLOYD, 2017).

Neste sentido, cita-se que os enfoques na Estratégia como processo são formulação e implementação, decisão e mudança. Burgelman (1996) defende a Estratégia como processo apontando que as variáveis da pesquisa em processo fornecem janelas para a "caixa preta" da criação de estratégias em organizações complexas. O autor posiciona-se a favor de abordagens positivo-descritivas para identificar e explicar paradoxos, círculos viciosos, dilemas e tensões no processo de formulação de estratégias que derivam das atividades de gerentes que estão diferentemente situados na organização e respondem a diferentes pressões externas e internas (BURGELMAN, 1996).

Sobre a importância da decisão, deve-se compreender, na EPO, que estas formam as estruturas e processos organizacionais (MINTZBERG, 1978). Este pensamento, segundo Mintzberg (1978), traduz a importância da Estratégiano processo, percebendo-a como orientadora das decisões organizacionais através de diretrizes deliberadas de forma consciente. Sobre a perspectiva teórico-deliberada, enfatiza-se a tendência mais formalista e preditiva. Whittington (1996) explica, nesse sentido, a proximidade do processo deliberado a uma escola clássica de abordagem teleológica e que está mais voltada ao planejamento.

No entanto, não é apenas a escola clássica que se destaca no contexto de estudos da EPO. Whittington (1996) identificou que o processo de formação de Estratégia poderia ser dado por quatro abordagens genéricas. Na abordagem clássica, há um processo racional e deliberado visando à maximização de lucros. A abordagem evolucionária apresenta-se em uma perspectiva de aplicação das noções da teoria da evolução à dinâmica das populações organizacionais. Neste 
contexto, enfatiza-se a naturalidade da estratégia como processo emergente, uma vez que as mudanças são imprevisíveis. A perspectiva emergente apresenta uma tendência incrementalista.

Na escola processual, Whittington (1996) destaca a metáfora do ciclo de vida. Neste sentido, dá maior ênfase à experiência e, não obstante o fato de considerar uma racionalidade limitada acredita que as forças de mercado também são imperfeitas. Desse modo, conforme destacaram Gimenez et al. (1999), os resultados são influenciados especialmente por questões ligadas a poder e cultura - o que pode restringir a maximização do lucro.

$\mathrm{Na}$ abordagem sistêmica, o foco volta ao racional em uma perspectiva deliberada que permite formular estratégias globais. Assim, admite uma análise ambiental e interpretativa e considera que aspectos subjetivos podem ser conflitantes com o lucro. Desse modo, desperta para necessidade da pluralidade nos objetivos para além da lucratividade (WHITTINGTON, 1996; WHITTINGTON et al., 2003).

De forma geral, pode-se dizer que, na Estratégia como processo, o processo é visto como a causa histórica do desempenho. De forma ampla, diz-se que a tentativa de criar teorias processuais gira em torno de observar as repetições sequenciais no tempo capazes de causar generalidades. Quanto a isto, se por um lado, a EPO fornece uma visão mais concreta, uma vez que a ação rastreia o fluxo de acontecimentos; por outro lado, a EPO acaba sendo criticada ao utilizar abordagens metodológicas que visam à generalização, fazendo com que, em um nível amplo, a performance seja concebida de forma idêntica àquela buscada pela Estratégia como conteúdo.

\subsection{Estratégia como Prática}

Com base na ideia seminal de que o sujeito nunca vai para a ação isento e que tal falta de neutralidade é recursiva - numa dinâmica na qual o jeito das pessoas influencia as práticas e as práticas influenciam o jeito das pessoas - nasce a ideia de estudar a Estratégia como prática (EPA) (VAARA; WHITTINGTON, 2012). No contexto da Estratégia como prática, os outcomes são mutuamente constitutivos em relação à práxis, e não dela separados por relações de causa-e-efeito.

Vaara e Whittington (2012) atribuem as origens da perspectiva da prática a Wittgenstein ou Heidegger, mas destacam o que chamam "virada prática" no social (c.f. RASCHE; CHIA, 2009), que surgiu graças à proliferação de pesquisas baseadas em teorias da prática (e.g. SEIDL; WHITTINGTON, 2014). Esse grupo de autores do campo da Estratégia destaca a contribuição de filósofos da era pós-estruturalista, como Foucault; de sociológicos como de Certeau e 
Giddens; antropólogos como Bourdieu; etnometodologistas como Garfinkel; teóricos da atividade como Engeström e Vygotsky; e estudiosos do discurso, como Fairclough.

No contexto de Estratégia como prática, é necessário compreender que a prática vai além do que se concebe como "prático"(FELDMAN; WORLINE, 2016). Há, então, uma ligação profunda entre a Estratégia e demais tradições de pesquisas em outras disciplinas. Vaara e Whittington (2012) atribuem às primeiras raízes das pesquisas em estratégia como prática o estudo de Knights e Morgan (1991) intitulado "Estratégia Corporativa, Organizações e Subjetividade: Uma Crítica" e o artigo de Whittington intitulado "Estratégia como Prática" (WHITTINGTON, 1996).

No cerne da EPA está o esforço de olhar as raízes culturais que direcionam as pessoas. Dito de outro modo há um olhar sociológico da estratégia nos campos organizacionais (WHITTINGTON, 2007). Essa profundidade no olhar estratégico destaca os benefícios da aplicação da Estratégia como prática. Assim, é possível um direcionamento maior a quem realmente administra as estratégias, oferecendo um nível de maior profundidade analítica capaz de oferecer mecanismos explicativos para campos padrões temporais observados (JOHNSON et al., 2007).

A complexidade da EPA pode também expandir as observações para o nível macro de análise e fornecer diversos direcionamentos aos pesquisadores. Whittington (2007) destaca que a EPA, tradicionalmente, foca em práticas e praticantes da Estratégia. Desse modo, pensar em Estratégia como prática é buscar, também, entendê-la como movimento que valoriza o fluxo sobre formas de fazer as coisas, enfatizando atores e rotinas que são compartilhadas ao longo do tempo (VAARA; WHITTINGTON, 2012; GOLSORKHI et al., 2015).

O rótulo de prática na Estratégia remete a uma tentativa de estar perto do mundo dos praticantes ao passo que estabelece um compromisso com as teorias sociológicas da prática (VAARA; WHITTINGTON, 2012). O modus operandi da Estratégia como prática, tem a performance como práxis. Neste sentido, convém situar a práxis no cerne da elaboração de estratégias. Vaara e Whittington (2012) destacam que um dos pontos fortes da abordagem é aprofundar o que realmente está acontecendo. Assim, pode-se compreender melhor o foco nos microprocessos em que a Estratégia como prática se dá (JOHNSON; MELIN; WHITTINGTON, 2003).

Contudo, para além do registro do que acontece no dia-a-dia, a práxis envolve a mútua constituição com a prática, de modo que a práxis e a prática são interdependentes. Whittington (2007) posiciona a questão da práxis de Estratégia sob um viés de suas atividades, o que justifica metodologias de estudos mais minuciosos como etnografia(BALOGUN; HUFF; 
JOHNSON, 2003). O apelo para um olhar sociológico para a Estratégia embasa a definição de que esta é uma prática social que, como casamento ou guerra, tem diversas facetas e ramificações (WHITTINGTON, 2007).

Alinhada à perspectiva de Whittington, está a ideia de Jarzabkowski, Balogun e Seidl (2007). Esses autores situam a EPA como composta de comportamentos rotineiros que englobam diversos elementos interligados entre si. Dentre estes elementos, estão as formas de atividades mentais e corporais; as relações entre atores, coisas e utilização; e os estados emocionais que incluem a motivação (JARZABKOWSKI; BALOGUN; SEIDL, 2007). O trabalho de Wenzel e Koch (2018) destaca que a literatura sobre Estratégia como prática concentra-se em explorar aspectos da elaboração de estratégias pelo desempenho do corpo. Porém, em uma perspectiva mais ampla, é possível estender a compreensão às práticas discursivas e como os atores se comunicam envolvendo elementos humanos e nãohumanos(BALOGUN et al., 2014).

Nesta linha de pensamento, entende-se que o discurso constitui parte significativa no trabalho da Estratégia como prática. Como observado por Wenzel e Koch (2018), os estrategistas têm investido cada vez mais tempo na preparação de uma comunicação estratégica por formas institucionalizadas de discursos. Neste processo, é fundamental considerar as ferramentas tecnológicas como facilitadoras da Estratégia, bem como abordagens metodológicas mais detalhadas, como supracitado.

Jarzabkowski, Balogun e Seidl (2007) identificam os praticantes como atores que, através de quem são, formas como agem e os recursos que utilizam, são capazes de dar forma à construção da prática. É uma discussão sobre o papel do agente e quem é este agente. Isso coloca uma dualidade entre estrutura e agência, além de focar em ações do dia-a-dia de forma mais prática pela ação na práxis.

Contudo, Splitter e Seidl (2011) vão desenvolver um ponto de vista que coloca em questão a aplicação prática da EPA em contextos de gestão. Splitter e Seidl (2011) propõem uma discussão sobre a relevância prática e o rigor científico das pesquisas focadas em prática. Eles criticam a formação da práxisna Estratégia, discutindo possibilidades de análise nos dois campos. Esses autores utilizam a perspectiva de Bourdieu para afirmar que apenas quando os estudos da EPA desenvolvem reflexividade é que têm o potencial de gerar conhecimento relevante. Ainda assim, a relevância não é instrumental, mas conceitual (SPLITTER; SEIDL, 2011).

\section{DISCUSSÃO}


A publicação de Whittington (2007) discute pontos diversos que implicam a Estratégia como processo e a estratégia como prática. Ele aponta que existe uma confusão ao contextualizar o tipo de tradição estratégica em questão e defende que esta confusão é compreensível uma vez que ele enxerga proximidade entre essas duas abordagens, que se unem ao se posicionarem como alternativas à ênfase do mainstream representado pela Estratégia como conteúdo.

Neste tópico será apresentada uma discussão que propõe um diálogo entre recentes propostas para a reflexão sobre a relação entre processos e práticas na Estratégia: Whittington (2007), Jarzabkowski et al. (2016), Burgelman et al. (2018) e outras fontes. As três discussões principais complementam-se no sentido de distinção entre EPA e EPO (WHITTINGTON, 2007); necessidade de vinculação dos três aspectos (conteúdo, processo e prática social) na prática gerencial (JARZABKOWSKI et al., 2016); e possibilidades de interseção e diálogo entre prática e processo (BURGELMAN et al., 2018).

"Estratégia como Prática não é Conteúdo, não faz muita Variação e provavelmente não é Substância; então deve ser o Processo" (WHITTINGTON, 2007, p. 1576). As palavras utilizadas nesse trecho devem-senão há concordância do autor com a afirmação e sim, a uma conclusão comum que costuma originara confusão sobre o escopo das pesquisas em processo e em prática.

Para Whittington, a causa fundamental da confusão entre prática e processo está na natureza dicotômica da literatura que pode, muitas vezes, situar a EPA como subordinada à tradição processual. Quanto a isto, é fundamental a observação de que o escopo da EPO não é tão amplo assim. Whittington justifica essa afirmação citando Mohr (1982) e Rescher (1996) que compreendem o "processo" como sendo a pesquisa sobre como e porque as coisas evoluem ao longo do tempo. Destaca-se, assim, a preocupação primordial com a evolução temporal das organizações, a qual não é o foco da EPA.

A respeito das questões que poderiam causar confusão em relação aos termos, a maior contribuição de Whittington (2007) foi sugerir um olhar sociológico para a Estratégia como característica principal da EPA. Desse modo, observando a prática com um olhar social, as pesquisas podem contribuir para discutir aspectos sociais da Estratégia que vão além do desempenho organizacional (VAARA; DURAND, 2012), o qual continua sendo o foco da corrente de processo. Contudo, deve-se observar que realizar a Estratégia como prática requer também olhar além dos modelos essenciais que se concentram apenas nas "melhores" práticas isoladamente, uma vez que estes podem apresentar práticas como fenômenos técnicos e 
abstratos, sem atentar para as dimensões sociais e historicamente localizadas de sua performance (JARZABKOWSKI et al., 2016).

Nessa tendência, Whittington (2007) destaca o que ele chama de "4 Os" da pesquisa em Estratégia: práxis, práticas, profissionais e profissão. Ele acrescenta a esse mix especialmente o posicionamento da Estratégia como um campo profissional, pois, para além das empresas, o campo inclui escolas de negócios, revistas de gestão, mídia, consultorias, gurus gerenciais etc. Na visão deste autor, nesses quatro pontos, há uma possibilidade de sobreposição em EPO e EPA apenas na práxis.

Assim, uma vez que a práxisde Estratégia volta-se aos outcomes, incluindo o desempenho econômico, neste nível poderia haver confusão quanto às abordagens. Aqui, podese citar a discussão de Splitter e Seidl (2011) que parecem concordar com esta sobreposição ao examinar a Estratégia nos contextos acadêmicos e de gestão. Acerca disto, é necessário buscar a reflexividade para prática e compreendê-la além do que é prático/pragmático em si.

Jarzabkowski et al. (2016) propõem um modelo integrativo que vai além do que interessa aos gestores em prática. Assim, além de responder quais práticas são importantes, os autores apresentam a questão de quem aplica e transfere as práticas; como as práticas são postas no campo; e depois integram o "o quê", o "quem" e o "como" prática da Estratégia. Este esforço integrativo atende tanto a gestores e consultores, quanto fornece diretrizes para uma pesquisa mais abrangente em nível acadêmico.

Para Whittington (2007), as características dos trabalhos pioneiros de Mintzberg sobre Estratégia colaboram para delimitar os limites entre EPO e EPA. O nível da práxis, apontado como passível de sobreposição nas duas abordagens, é diminuído na EPO graças ao seu foco nos resultados organizacionais e marginalização das práticas quem têm sua utilidade em si.

Os autores Lechner e Müller-Stewens (2000) apontam que a Estratégia como processo levou a um foco teórico limitado. Como a ênfase era colocada nas decisões, a pesquisa precisava excluir todos os outros fenômenos organizacionais que não podiam ser conceituados como decisões. No entanto, nem todos os eventos que influenciam a formação de estratégias são decisões explícitas.

Questões como decisões, predominantes no processo, podem dar base para a EPA, porém esta, segundo Whittington (2007), seria mais próxima a um status de prima da EPO do que de irmã, uma vez que focar nas decisões como base para o desempenho seria minimizar ou mesmo negar a complexidade das relações que Whittington insiste serem possíveis de se enxergar apenas com um olhar sociológico. 
Em um esforço para oferecer uma abordagem crítica autoconsciente quanto ao papel da Estratégia, também se destacam Knights e Morgan (1991), que criticam abordagens estratégicas processualistas que não enxergam os micro processos, nem vêem o discurso como uma problemática. Quanto a isso, pode-se citar o trabalho de Burgelman et al. (2018) que propõe uma combinação entre as abordagens estratégicas processuais e práticas. $\mathrm{O}$ autor reconhece que as análises discursivas e narrativas no sentido de uma comunicação estratégica constituem um tópico pouco explorado na Estratégia como processo, mas sugerem que chegou o momento de combinar percepções das várias tradições discursivas e narrativas em ambas as áreas a fim de enriquecer nossa compreensão acerca das dinâmicas e práticas da comunicação estratégica em vários contextos.

A partir da análise de Burgelman et al. (2018), é possível compreender o papel das narrativas estratégicas nos processos de longo prazo, o que tornaria a pesquisa concreta permitindo o surgimento de ideias estratégicas. Os autores citam como exemplo o estudo de Vaara, Sonenshein e Boje (2016). Nesse estudo, os autores sustentam que, não obstante os avanços de análises narrativas nos estudos organizacionais há ainda um desvio do foco do papel das narrativas. Visando estabilidade e mudança organizacional, os autores fornecem uma estrutura conceitual mais profunda para a análise narrativa.

Neste sentido, aprofundar a análise narrativa constitui um desafio para aprofundar a visão processual. Cita-se ainda que, na abordagem de prática, há diferenças quanto à inserção metodológica no campo, o que pode ser benéfico para compreensão dos arranjos micro das atividades organizacionais (c.f. BALOGUN; HUFF; JOHNSON, 2003). Estas diferenças se concentram nos esforços para desenvolver estudos de base sociológica como etnografias.

Ao que se percebe, o estudo da Estratégia, em qualquer abordagem, parece concordar que a realização estratégica e a sua manutenção necessitam de rotinas, procedimentos e pessoas para executá-las. Nesse aspecto, não seria precipitado concordar com Whittington (2007) quando explica que a Estratégia demanda uma visão completa sob o olhar sociológico uma vez que deve se preocupar com a compreensão das conexões da Estratégia, bem como com sua inserção e, por conseguinte, as mudanças e continuidades organizacionais (WHITTINGTON, 2018).

Para além de todas as coisas que compõem a Estratégia, Burgelman et al. (2018) identificam cinco correntes de pesquisa acerca da EPA. Assim, de forma mais ampla, deve-se pensar: nas práticas sócio-organizacionais na construção de estratégias; nos papéis e identidades de quem pratica a estratégia; no discurso e na abordagem do sensemaking e narrativas; na 
sociomaterialidade e ferramentas de Estratégia; além das questões de poder e de reflexão crítica sobre o status quo.

A EPA fundamenta-se na pesquisa das práticas sociais e organizacionais. A característica multinível da Estratégia como prática faz com que ela seja incorporada nas rotinas, operações e cultura das organizações. Contudo, essa visão micro fundamenta uma das críticas sobre a pesquisa em Estratégia como prática. Diante das limitações e possibilidades entre a EPA e EPO, Burgelman et al. (2018) destacam argumentos apaziguadores para combinar as abordagens.

A visão complementar entre EPO e EPA entende que cada abordagem examina fenômenos que, embora diferentes, são compatíveis. Desse modo, a visão profunda em contexto micro possibilitada pela EPA estaria dentro de processos maiores, abordados pela EPO. A segunda visão é a visão crítica, que propõe uma separação entre prática e processo. Se por um lado, os defensores da EPA apontam uma deturpação da visão processual sobre o micro, por outro lado, os defensores da Estratégia como processo assumem um ceticismo sobre o que é relevante da corrente da prática para o dia a dia da Estratégia.

Burgelman et al. (2018) concordam, então, com uma terceira via, uma visão combinatória que visualiza o entrelaçamento entre atividades e processos em um mesmo fenômeno. Segundo esses autores, nesta visão residem as maiores oportunidades de desenvolvimento de pesquisas conjuntas e podem ser sintetizadas em um corpo único e coerente de pesquisa. De modo geral, os autores perceberam que, em uma ontologia mais forte de processos, o processo refere-se à atividade contínua e, assim, aproxima-se da ênfase historicista da EPA. Desse modo, há simultaneidade nas atividades que reformam as organizações e as reproduzem com o tempo, voltando-se para análise dos macro fenômenos que as envolvem (JARZABKOWSKI, 2004). Do mesmo modo, as práticas dependem da continuidade para ampliar o alcance, o que fortalece o argumento de olhar para os microfenômenos.

\section{CONCLUSÃO}

Após expor diferentes perspectivas sobre a questão da Estratégia, é possível perceber um ponto filosófico convergente entre as abordagens: ambas, a EPO e EPA, admitem que as organizações seguem fluxos em movimentos. Desse modo, convém refletir sobre se existem ainda lacunas nas quais a Estratégia ainda não foi explorada.

É comum no mainstream das disciplinas adotar posturas defensivas quanto a diferentes epistemologias. Contudo, pode-se levar adiante a possibilidade de conciliação em uma 
perspectiva combinatória, como propôs Burgelman et al. (2018) ou compartilhar das reticências em forma provocativa como tentaremos aqui. As propostas para combinação foram exploradas e aparentemente apaziguam os ânimos sobre as lacunas ainda existentes. De modo particular, no entanto, defendemos que o embate é mais profundo do que a discussão sobre adequação de diferentes abordagens de Estratégia umas às outras.

Aqui propomos que talvez exista uma disputa entre campos. De um lado, a perspectiva da gestão, mais voltada aos interesses econômicos e, de outro, a perspectiva sociológica, interessada nos diferentes discursos e contextos sociomateriais que compõem a vida cotidiana. Neste aspecto, talvez se possa sugerir que, enquanto a questão do imbricamento da organização na sociedade for percebida de forma crítica, apontando para conflitos de interesses, valores e crenças entre entes sociais, não há, em curto prazo, uma possibilidade combinatória com os interesses que focam no desempenho de mercado.

Enquanto a possibilidade combinatória no âmbito gerencial pode ser alcançada por uma análise ontológica forte do processo, a possibilidade de análise ontológica forte do agente está distante de ser alcançada. Desse modo, compreendemos que o posicionamento da Estratégia como processo está mais para o campo da gestão, enquanto o posicionamento da Estratégia como prática está mais para o campo sociológico. Portanto, em suma, essa clivagem entre processo e prática social no campo da Estratégia parece refletir, de forma mais ampla, a proeminência do mercado e de sua lógica analítico-racional, de um lado, ou da sociedade e de sua construção político-institucional, de outro, como o verdadeiro pano de fundo das organizações. Obviamente, tal posicionamento tem implicações diretas para a pluralidade da pesquisa, tanto em questões metodológicas quanto em implicações gerenciais. Tentativas de integrações abrangentes podem ser interessantes, mas, dadas as crenças e os valores fundamentalmente distintos que subjazem a essas abordagens, o que parece permanecer no momento em relação ao futuro de possibilidades para essas tradições e sua combinação são reticências - mais do que pontos finais.

\section{REFERÊNCIAS}

ARGYRES, N.; MCGAHAN, A. M. Introduction: Michael Porter's competitive strategy. Academy of Management Executive, v. 16, n. 2, p. 41-42, 2002.

BALOGUN, J. et al. Placing strategy discourse in context: sociomateriality, sensemaking, and power. Journal of Management Studies, v. 51, n. 2, p. 175-201, 2014.

BALOGUN, J.; HUFF, A. S.; JOHNSON, P. Three responses to the methodological challenges of studying strategizing. Journal of Management Studies, v. 40, n. 1, p. 197-224, 2003. 
BARNEY, J. Firm resources and sustained competitive advantage. Journal of Management, v. 17, n. 1, p. 99-120, 1991.

BURGELMAN, R. A. A Process model of strategic business exit: implications for an evolutionary perspective on strategy. Strategic Management Journal, v. 17, n. S1, p. 193 214, 1996.

BURGELMAN, R. A. et al. Strategy processes and practices: dialogues and intersections. Strategic Management Journal, v. 39, n. 3, p. 531-558, 2018.

CHIA, R. Essai: time, duration and simultaneity: rethinking process and change in organizational analysis. Organization Studies, v. 23, n. 6, p. 863-868, 2002.

CHIA, R.; MACKAY, B. Post-processual challenges for the emerging strategy-as-practice perspective: discovering strategy in the logic of practice. Human Relations, v. 60, n. 1, p. 217-242, 2007.

FELDMAN, M.; WORLINE, M. The practicality of practice theory. Academy of Management Learning \& Education, v. 15, n. 2, p. 304-324, 2016.

FLOYD, S. W. et al. Processes and practices of strategizing and organizing: review, development, and the role of bridging and umbrella constructs. Journal of Management Studies, v. 48, n. 5, p. 933-952, 2011.

FREITAS, J. et al. Perspectivas teóricas do mainstream da administração estratégica: uma meta-síntese. Revista Inteligência Competitiva, Santana de Parnaíba v. 7, n. 1, p. 154-182, 2017.

GIMENEZ, F. A. P. et al. Estratégia em pequenas empresas: uma aplicação do modelo de Miles e Snow. Revista de Administração Contemporânea, Curitiba, v. 3, n. 2, p. 53-74, 1999.

GOLSORKHI, D. et al. Introduction: what is strategy as practice? In. GOLSORKHI, D. et al. (Org.). Cambridge handbook on strategy as practice. Cambridge, England: Cambridge University Press, p. 1-29, 2015.

GRECKHAMER, T. The stretch of strategic management discourse: a critical analysis. Organization Studies, v. 31, n. 7, p. 841-871, 2010.

GUERRAS-MARTÍNA, L. Á.; MADHOKB, A.; MONTORO-SÁNCHEZC, Á. The evolution of strategic management research: recent trends and current directions. BRQ Business Research Quarterly, v. 17, n. 2, p. 69-76, 2014.

HERRMANN, P. Evolution of strategic management: the need for new dominant designs. International Journal of Management Reviews, v. 7, n. 2, p. 111-130, jun. 2005.

HOSKISSON, R. E. et al. Theory and research in strategic management: swings of a pendulum. Journal of Management, v. 25, n. 3, p. 417-456, 1999.

HUFF, A. S.; REGER, R. K. A review of strategic process research. Journal of Management, v. 13, n. 2, p. 211-236, 1987. 
HUTZSCHENREUTER, T.; KLEINDIENST, I. Strategy-process research: what have we learned and what is still to be explored. Journal of Management, v. 32, n. 5, p. 673-720, 2006.

JARZABKOWSKI, P. Strategy as practice: recursiveness, adaptation, and practices-in-use. Organization Studies, v. 25, n. 4, p. 529-560, 2004.

JARZABKOWSKI, P.; BALOGUN, J.; SEIDL, D. Strategizing: the challenges of a practice perspective. Human Relations, v. 60, n. 1, p. 5-27, 2007.

JARZABKOWSKI, P.; KAPLAN, S. Taking "strategy-as-practice" across the Atlantic. In: BAUM, J. A. C.; LAMPEL, J. (Ed.). Globalization of strategy research. Bingley-UK: Emerald Publishing, 2010. p. 51-71. (Advances in Strategic Management, 27).

JARZABKOWSKI, P. et al. If you aren't talking about practices, don't call it a practice-based view: rejoinder to bromiley and rau in strategic organization. Strategic Organization, v. 14, n. 3, p. 270-274, 2016.

JOHNSON, G. et al. Strategy as practice: research directions and resources. Cambridge: Cambridge University Press, 2007.

JOHNSON, G.; MELIN, L.; WHITTINGTON, R. Micro strategy and strategizing: towards an activity-based view. Journal of Management Studies, v. 40, n. 1, p. 3-22, 2003.

KNIGHTS, D.; MORGAN, G. Corporate strategy, organizations, and subjectivity: a critique. Organization Studies, v. 12, n. 2, p. 251-273, 1991.

KOUAMÉ, S.; LANGLEY, A. Relating microprocesses to macro-outcomes in qualitative strategy process and practice research. Strategic Management Journal, v. 39, n. 3, p. 559$581,2018$.

LANGLEY, A. Process thinking in strategic organization. Strategic organization, v. 5, n. 3, p. 271-282, 2007.

LECHNER, C.; MÜLLER-STEWENS, G. et al. Strategy process research: what do we know, what should we know? In: DAHIYA, S. B. (Ed.). The current state of business disciplines: v. 4 Management. Rohtak: Spellbound Verlag, 2000. p. 1863-1893.

MELLAHI, K.; SMINIA, H. Introduction: the frontiers of strategic management research. International Journal of Management Reviews, v. 11, n. 1, p. 1-7, 2009.

MINTZBERG, H. Patterns in strategy formation. Management Science, v. 24, n. 9, p. 934948, 1978.

MINTZBERG, H; WATERS, J A. Of strategies, deliberate and emergent. Strategic Management Journal, v. 6, n. 3, p. 257-272, 1985.

MOHR, L. B. Explaining organizational behavior. San Francisco (CA-US): Jossey-Bass, 1982. 
PRAHALAD, C. K.; HAMEL, G. The core competence of the corporation. Harvard Business Review, v. 68, n. 3, p. 79-91, 1990.

PORTER, M. E. O que é estratégia. Harvard Business Review, v. 74, n. 6, p. 61-78, 1996.

RASCHE, A.; CHIA, R. Researching strategy practices: a genealogical social theory perspective. Organization Studies, v. 30, n. 7, p. 713-734, 2009.

RESCHER, N. Process metaphysics: an introduction to process philosophy. New York: State University of New York Press, 1996.

SEIDL, D.; WHITTINGTON, R. Enlarging the strategy-as-practice research agenda: towards taller and flatter ontologies. Organization Studies, v. 35, n. 10, p. 1407-1421, 2014.

SMINIA, H. Process research in strategy formation: theory, methodology and relevance. International Journal of Management Reviews, v. 11, n. 1, p. 97-125, 2009.

SPANOS, Y. E.; LIOUKAS, S. An examination into the causal logic of rent generation: contrasting porter's competitive strategy framework and the resource-based perspective. Strategic Management Journal, v. 22, n. 10, p. 907-934, 2001.

SPLITTER, V.; SEIDL, D. Does practice-based research on strategy lead to practically relevant knowledge? Implications of a Bourdieusian perspective. The Journal of Applied Behavioral Science, v. 47, n. 1, p. 98-120, 2011.

STONEHOUSE, G.; SNOWDON, B. Competitive advantage revisited: Michael Porter on strategy and competitiveness. Journal of Management Inquiry, v. 16, n. 3, p. 256-273, 2007.

TEECE, D. J. Explicating dynamic capabilities: the nature and microfoundations of (sustainable) enterprise performance. Strategic Management Journal, v. 28, n. 13, p. 13191350, 2007.

TEECE, D.J.; PISANO, G.; SHUEN, A. Dynamic capabilities and strategic management. Strategic Management Journal, v. 18, n. 7, p. 509-533, 1997.

VAARA, E.; DURAND, R. How to connect strategy research with broader issues that matter? Strategic Organization, v. 10, n. 3, p. 248-255, 2012.

VAARA, E.; WHITTINGTON, R. Strategy-as-practice: taking social practices seriously. Academy of Management Annals, v. 6, n. 1, p. 285-336, 2012.

VAARA, E.; SONENSHEIN, S.; BOJE, D. Narratives as sources of stability and change in organizations: approaches and directions for future research. Academy of Management Annals, v. 10, n. 1, p. 495-560, 2016.

WENZEL, M.; KOCH, J. Strategy as staged performance: a critical discursive perspective on keynote speeches as a genre of strategic communication. Strategic Management Journal, v. 39, n. 3, p. 639-663, 2018. 
WHITTINGTON, R. Strategy as practice. Long Range Planning, v. 29, n. 5, p. 731-735, 1996.

WHITTINGTON, R. Completing the practice turn in strategy research. Organization Studies, v. 27, n. 5, p. 613-634, 2006.

WHITTINGTON, R. Strategy practice and strategy process: family differences and the sociological eye. Organization Studies, v. 28, n. 10, p. 1575-1586, 2007.

WHITTINGTON, R. Greatness takes practice: on practice theory's relevance to "great strategy". Strategy Science, v. 3, n. 1, p. 343-351, 2018.

WHITTINGTON, R. et al. Taking strategy seriously: responsibility and reform for an important social practice. Journal of Management Inquiry, v. 12, n. 4, p. 396-409, 2003.

WOLF, C.; FLOYD, S.W. Strategic planning research: toward a theory-driven agenda. Journal of Management, v. 43, n. 6, p. 1754-1788, 2017.

\section{DADOS DOS AUTORES}

\section{Hellen Taynan da Silva Cavalcanti}

Currículo Lattes: http://lattes.cnpq.br/0462162780986588

E-mail: hellentaynan@gmail.com

Doutoranda em Administração no CEPEAD na Universidade Federal de Minas Gerais. Mestre em Administração e Sociedade com ênfase em Marketing e Sociedade no Programa de PósGraduação da Universidade Federal da Paraíba (2017). Formação livre em Psicanálise pelo Instituto Internacional de Terapia (2013). Especialista em Administração e Marketing pelo Grupo Uninter (2012). Graduada em Administração pela Universidade Federal de Pernambuco (2010). Membro do Grupo de Pesquisas GP CIBER na UFPB desde 2014.

\section{Jonathan Simões Freitas}

Currículo Lattes: http://lattes.cnpq.br/5394006847919001

E-mail: prof.jonathanfreitas@gmail.com

Pós-doutorado (PDJ/CNPq) em Administração (2016), na área de Metodologia de Pesquisa, no CEPEAD. Doutor (2014) em Administração (UFMG), na área de Estratégia, com período sanduíche na École des Hautes Études Commerciales (HEC) Montréal/Canadá. Mestre (2009) em Administração (UFMG), na área de Estratégia. Graduado (2007) em Engenharia de Produção (UFMG), com estudos na ESIEE Amiens/França. Professor adjunto do Departamento de Ciências Administrativas (CAD) e docente permanente do Centro de Pós-Graduação e Pesquisas em Administração (CEPEAD) da Faculdade de Ciências Econômicas (FACE) da Universidade Federal de Minas Gerais (UFMG) na área de Administração Geral (Estratégia). Pesquisador do Núcleo de Ensino e Pesquisa em Mercadologia e Estratégia em Organizações (NUME), do Núcleo de Tecnologia da Qualidade e da Inovação (NTQI) e do Observatório de Logística Urbana e Mobilidade (LOG-URB) da UFMG. Representante titular da FACE no Comitê de Ética em Pesquisa (COEP) da UFMG. 